\title{
Panorama atual e perspectivas em relação ao diagnóstico precoce do câncer de ovário
}

\author{
Current situation and new perspectives on the early diagnosis \\ of ovarian cancer
} Montreal QC H2P 1E2 - Canadá
A prevalência mundial do câncer de ovário é de cerca de meio milhão de mulheres em um período de cinco anos; aproximadamente outros 200.000 novos casos ocorrem a cada ano ${ }^{1}$. O câncer de ovário é a neoplasia ginecológica mais letal e a sétima causa mais comum de óbito em mulheres. Menos da metade das mulheres (43\%) sobrevivem por mais de cinco anos após o diagnóstico de câncer de ovário, enquanto que os cânceres de endométrio e colo do útero estão associados a sobrevidas em cinco anos de, respectivamente, 83 e $72 \%$. Esses resultados desfavoráveis são, em boa medida, explicados pelo diagnóstico em geral tardio das neoplasias ovarianas: cerca de $70 \%$ dos casos são detectados quando estão nos estádios III e IV, para os quais a sobrevida média livre de doença não ultrapassa 18 meses². $^{2}$.

A incidência do câncer de ovário é mais elevada nos países industrializados, embora concentre o maior número de casos (96.700 versus 107.500) nos países em desenvolvimento. $\mathrm{Na}$ América Latina, a incidência de 8/100.000 mulheres aproxima-se daquela dos países desenvolvidos, que é de 10/100.000 mulheres. Nos países em desenvolvimento como um todo, no entanto, a incidência é mais baixa: 5/100.000. No Brasil, a incidência supera a dos países industrializados em dois registros de câncer de base populacional: Porto Alegre $\left(13 / 100.000\right.$ mulheres) e São Paulo $(11 / 100.000)^{1}$. O risco de vir a ter câncer de ovário aumentou de 1:70, em 1970, para 1:55, em 2006, embora fosse esperada certa redução da incidência dada à rápida disseminação, a partir da década de 1960, do uso de anovulatórios hormonais, os quais têm efeito comprovadamente protetor ${ }^{3}$.

Nos últimos 20 anos, a sobrevida das mulheres com câncer de ovário aumentou em dois anos ${ }^{4}$; este fato não se deveu ao diagnóstico mais precoce da doença, mas a esquemas de quimioterapia baseada em platina, introduzidos na década de 1980, e ao uso de taxanes, a partir da década de $1990^{2}$. Vale notar que esta melhora se deu em mulheres sem possibilidades de cura, enquanto a erradicação completa da doença, no mesmo período, aumentou apenas de 12 para $14 \%{ }^{2}$.

Em cerca de $90 \%$ dos casos, o carcinoma epitelial de ovário acomete mulheres com mais de 50 anos de idade, enquanto apenas os $10 \%$ restantes são diagnosticados em mulheres com síndromes hereditárias que, na maioria das vezes (65 a 75\%), são decorrentes de mutações nos genes BRCA1 e BRCA2 ${ }^{4}$. Nesses casos, o diagnóstico costuma ocorrer em idades menos avançadas. Em contraste, na população geral, a idade média ao diagnóstico é de 63 anos. O perfil epidemiológico típico para risco de câncer de ovário é o da mulher após a menopausa, nulípara, de nivel socioeconômico mais elevado ${ }^{4}$.

Faculdade de Ciências Médicas da Universidade Estadual de Campinas - UNICAMP - Campinas (SP), Brasil; Departamento de Obstetrícia e Ginecologia da Universidade McGill - Canadá

'Professora-associada do Departamento de Tocoginecologia da Faculdade de Ciências Médicas da Universidade Estadual de Campinas - UNICAMP - Campinas (SP), Brasil.

2 Professora-assistente do Departamento de Medicina Familiar da Universidade McGill e Conselheira Científica do Instituto Nacional de Saúde Pública de Quebec (INSPQ) - Montreal, Canadá.

3 Professor Doutor do Departamento de Tocoginecologia da Faculdade de Ciências Médicas da Universidade Estadual de Campinas - UNICAMP - Campinas (SP), Brasil. 
Embora outros procedimentos estejam sendo avaliados, a única recomendação para a prevenção primária da doença é a salpingo-ooforectomia bilateral (SOB) em pacientes portadoras de síndromes hereditárias, que estão sob risco elevado. Para mulheres que tenham herdado a mutação nos genes BRCA1 e BRCA2, a probabilidade de desenvolver a neoplasia é de 36 a $46 \%$ e de 10 a $27 \%$, respectivamente 5 .

Tradicionalmente, postulavam-se duas vias para a carcinogênese ovariana: uma relacionada aos ciclos ovulatórios e outra pela ação dos hormônios gonadotrópicos. Os estudos epidemiológicos e os experimentos biológicos desenvolvidos para testar essas teorias não foram conclusivos ${ }^{4}$. Atualmente, a biologia molecular, apoiada pelos recentes avanços em bioinformática, viabilizou estudos com ênfase em genômica, transcriptômica e proteômica, visando esclarecer a etiologia da doença. Muitos marcadores moleculares têm sido apontados como potencialmente relevantes à prevenção, ao diagnóstico e à terapêutica da doença. Mas vale lembrar que, embora a lista seja extensa, a exemplo de CA 125, HE4, mesotelina, osteopontina, HER-2, SMRP, CA72-4, IGFBP-3, EGFR e mucinas, entre outros, nenhum deles, exceto CA 125 , está validado para uso clínico ${ }^{6,7}$.

De especial interesse são as moléculas relacionadas ao processo inflamatório, a respeito das quais se acumulam evidências de participação na formação de tumores sólidos. Em 1999, Ness e Crotteau ${ }^{8}$ propuseram que os mecanismos inflamatórios "agiriam em combinação com, e em adição à ovulação e à ação dos hormônios gonadotróficos ao mediarem o risco para a doença”. Dois estudos recentes confirmam resultados anteriores, mostrando um aumento de risco associado ao uso prolongado de talco, história de endometriose e uso prolongado de anti-inflamatórios ${ }^{9,10}$. A constante reparação do epitélio após cada ovulação é a base da teoria da agressão inflamatória. Sabe-se que as condições associadas a um maior número de ovulações, tais como a menarca precoce, menopausa tardia e nuliparidade, são associadas ao risco de desenvolver a doença, assim como história de câncer de mama. Por outro lado, gestações a termo, lactação, uso de contraceptivo hormonal oral, ligadura tubária, histerectomia e uso de vitamina D conferem proteção. Não há evidências conclusivas sobre o aumento de risco relacionado ao índice de massa corpórea, à dieta pobre em vegetais ou rica em gordura, ao consumo de álcool e tabagismo e à exposição a dietilestilbestrol ou asbestos, entre outros fatores. Recentemente, surgiram estudos demonstrando a importância da história familiar de câncer de ovário: história positiva para mãe ou irmã duplica o risco para aparecimento em idades mais avançadas e quintuplica para diagnósticos antes dos 50 anos de idade, com maior tendência para tumores não mucinosos ${ }^{11}$.

A maioria dos tumores de ovário tem origem epitelial (mais de 80\%); 10 a 15\% são tumores de células germinativas e 5 a $10 \%$, tumores do estroma. Entre os tumores epiteliais, $40 \%$ são do tipo seroso, seguidos pelos tumores mucinosos, endometrioides, de células claras e mistos ${ }^{12}$. Entretanto, a classificação dos carcinomas de ovário não é simples, e a concordância interobservador é relativamente baixa ${ }^{12}$. Acreditava-se que os carcinomas de ovário originavam-se do epitélio ovariano, disseminando-se, a seguir, para as cavidades pélvica e abdominal. Entretanto, esse modelo de disseminação progressiva parece não se aplicar universalmente a todos os carcinomas ovarianos. Mais recentemente, baseando-se em dados clínicos, histopatológicos e de genética molecular, sugere-se agrupar os tumores ovarianos em apenas dois grupos, com comportamento biológico distinto, denominados tipos I e $\mathrm{II}^{13,14}$. Os tumores do tipo I tendem a ser diagnosticados no estádio I; são carcinomas bem diferenciados e se desenvolvem provavelmente a partir de lesões precursoras benignas. Estes incluem os carcinomas serosos micropapilares de baixo grau, mucinosos, endometrioide e de células claras. São de crescimento lento e, por isso, frequentemente detectados como grandes tumores anexiais restritos ao ovário. Os tumores do tipo II correspondem aos carcinomas indiferenciados que surgiriam diretamente do epitélio de superfície do ovário ou de cistos de inclusão, quase sempre diagnosticados em estádios avançados e apresentando um comportamento extremamente agressivo. Tumores do tipo II são representados por carcinomas serosos indiferenciados, mesodermais mistos e carcinomas indiferenciados. Essa classificação teria relevância para o planejamento das estratégias de prevenção da doença.

Quando a doença é diagnosticada em estádio I, ou seja, está restrita aos ovários, a sobrevida em cinco anos é de cerca de $94 \%$. A sobrevida é reduzida para $68 \%$ no estádio II e $30 \%$ para os estádios III e IV. Apenas $19 \%$ das mulheres americanas têm a doença diagnosticada em estádio I, enquanto que mais de $70 \%$ são diagnosticadas nos estádios III ou $\mathrm{IV}^{15}$. Outros países registram estatísticas semelhantes ${ }^{16}$. Nesse grupo de mulheres, os fatores de bom prognóstico são volume tumoral pequeno antes e depois da cirurgia, idade menor, bom estado geral, tumores bem diferenciados com tipo histológico outro que mucinoso ou de células claras, e ausência de ascite².

\section{Prevenção primária do carcinoma de ovário}

Para casos esporádicos, que constituem a maioria dos carcinomas de ovário, a estratégia de prevenção primária mais significativa é o uso de contraceptivos hormonais orais. Existe comprovada associação entre o uso desses contraceptivos e a redução da incidência da doença, acompanhada de efeito dose-resposta significativo: quanto mais prolongado o tempo de uso, maior a redução do risco de câncer. Essa proteção continua por pelo menos 30 anos após o término do uso ${ }^{17}$. 
Em uma outra revisão, foram analisados os dados de 45 estudos anteriormente publicados que, embora não fossem prospectivos, somam mais de 23.000 casos e 87.000 controles e utilizam metodologia apropriada, o que lhe confere robustez epidemiológica e estatística ${ }^{18}$. Não houve redução na frequencia de tumores mucinosos (12\% do total de casos), mas, para todos os demais tipos, houve redução semelhante. Estima-se, a partir dos dados desse estudo, que o uso de pílula anticoncepcional pela população de um país com alta incidência, por dez anos, cause uma redução de $30 \%$ na incidência e outros $35 \%$ na mortalidade pela doença. Calcula-se que o uso de anticoncepcional oral já tenha prevenido 100.000 óbitos pela doença e 200.000 casos e chegará a prevenir 30.000 casos por ano nas próximas décadas.

Outra estratégia preventiva seria a remoção cirúrgica dos ovários junto com a histerectomia por outras doenças uterinas. Entretanto, a remoção dos ovários não elimina completamente o risco de câncer de ovário e não existem estudos prospectivos que justifiquem essa conduta. Uma análise recente demonstrou que os efeitos deletérios da ooforectomia em mulheres na pré-menopausa ultrapassam os benefícios. A perda prematura da função ovariana antes da menopausa natural aumenta o risco de morte prematura, de doenças cardiovasculares, de deficiências cognitivas e demência, Parkinson, redução do bem-estar psicológico e diminuição da função sexual ${ }^{19}$. Essas consequências são, provavelmente, devidas a uma diminuição abrupta dos níveis de estrógenos, testosterona e progestágenos dos efeitos desta redução sobre o eixo hipotálamo-hipofisário, com consequente aumento das gonadotropinas ${ }^{19}$.

Para paciente comprovadamente portadora de mutação no gene BRCA1 ou BRCA2, recomenda-se preventivamente mastectomia e SOB, uma vez estabelecida sua prole. Uma metanálise recente confirma que, após SOB, há redução do risco para câncer de mama de $50 \%$, tanto para portadoras de mutações no BRCA1 quanto no BRCA2, e a redução de câncer de ovário e de tuba uterina de $80 \%$ (com intervalo entre 60 e 90\%). Não se recomenda o simples acompanhamento dessas mulheres com CA 125 sérico e ultrassonogafia pélvica transvaginal (UPTV) ${ }^{5,20}$.

\section{Rastreamento}

É importante que os esforços visando ao rastreamento do câncer de ovário resultem em redução da mortalidade. Por definição, testes de rastreamento devem detectar a doença em fases assintomáticas, permitindo a cura. É possível atingir ambos os objetivos, pois tumores ovarianos em estádio I têm, como mencionado anteriormente, alto potencial de cura. Há, porém, um obstáculo aqui, pois ainda não se identificou uma lesão precursora do câncer do ovário. Assim, não se pode testar a hipótese de que estas lesões sejam efetivamente diagnosticáveis e tratáveis, e, desta forma, prevenir a formação de tumores invasores e erradicar a doença antes de sua apresentação em forma invasora. Se, no entanto, a classificação histopatológica proposta por Kurman e Shih ${ }^{13}$ for correta, os tumores no estádio I são distintos daqueles mais agressivos, e o maior obstáculo para um programa de prevenção secundária será a possibilidade de lead time bias. Desta forma, seriam detectados tumores menos agressivos, sem que se obtenha impacto sobre a mortalidade.

Outra barreira importante é o desempenho insatisfatório dos testes hoje disponíveis, entre eles as medidas séricas de CA 125 e a UPTV. Um teste com desempenho aceitável para o rastreamento do câncer de ovário deveria oferecer especificidade acima de $99 \%$ e valor preditivo positivo mínimo de 10\%, ou seja, dez cirurgias para cada câncer diagnosticado (ou resultar em nove resultados falsos-positivos ${ }^{21}$. Não é preciso enfatizar a dificuldade para se obter tal teste. O desempenho insuficiente dos testes de que dispomos, CA 125 e UPTV, é decorrente da relativamente baixa prevalência da doença e da dificuldade em se lidar com resultados falsos-positivos, que invariavelmente levariam a explorações cirúrgicas desnecessárias.

Mais de $80 \%$ das mulheres com câncer de ovário avançado apresentam CA 125 elevado (>30 ou 35 U/mL). Entretanto, naquelas com doença inicial, ou seja, curável e que seriam o objetivo final do rastreamento, apenas 50 a $60 \%$ têm o teste positivo ${ }^{6}$. Tais valores estão aquém dos preconizados pela Organização Mundial da Saúde (OMS). O desempenho ideal não foi obtido nem mesmo combinando-se as dosagens de CA 125 com a UPTV ${ }^{6}$. Ademais, a acurácia da ultrassonografia depende de um examinador experiente: este leva em conta, inconscientemente, muitas variáveis demográficas e clínicas, além daquelas ultrassonográficas, o que faz com que nenhum modelo matemático tenha sido melhor, até hoje, do que a opinião de um ecografista especialista ${ }^{22}$. Consequentemente, essa característica do teste dificulta a implantação de um programa de base populacional.

De qualquer forma, apesar dessas muitas barreiras, vários grupos de pesquisadores conduziram estudos recrutando milhares de pacientes assintomáticas da população geral, mas dentro de certos outros critérios de elegibilidade - tais como idade acima de 45 ou 50 anos, sem história de SOB ou câncer - que foram submetidas a diferentes modalidades de rastreamento ${ }^{21}$. Resumidamente, estas modalidades incluíram algoritmos com medidas únicas ou seriadas de CA 125 sérico, UPTV ou ambos ao mesmo tempo ou em tempos diferentes. Jacobs et al. ${ }^{23}$ conseguiram obter um aumento da sobrevida média de $100 \%$ no grupo de intervenção (rastreamento com CA 125 seguido de UPTV) comparado ao grupo controle ( 73 versus 42 meses, $\mathrm{p}=0,011$ ), em um ensaio randomizado incluindo 22.000 participantes com sete anos de seguimento. Esses autores documentaram ainda um risco relativo de óbito duas vezes maior para o grupo 
controle $(\mathrm{RR}=2$; IC95\%=0,78-5,13) e argumentam que, embora não significativo estatisticamente, esse risco poderia representar ou ausência de diferença de mortalidade entre os grupos, ou uma diminuição da mortalidade de cinco vezes no grupo rastreado. Embora outros pesquisadores continuem céticos em relação à efetividade do rastreamento, estudos ainda maiores, baseados em estratégias semelhantes ou idênticas de rastreamento, foram conduzidos. Recentemente, terminou o recrutamento de mais de 220.000 mulheres em um ensaio randomizado na Inglaterra, além de 75.000 mulheres em outro, nos Estados Unidos, coordenado pelo National Institutes of Health Prostate, Lung, Colorectal, and Ovarian (PLCO) Cancer Screening Trial. Devido ao seguimento necessário após os testes iniciais, os resultados preliminares desses projetos só deverão ser divulgados após $2015^{24,25}$.

Com as evidências de que dispomos no momento e dado que nenhuma estratégia de rastreamento de câncer de ovário já avaliada tenha levado à redução da mortalidade, tanto a US Preventive Services Task Force quanto sua correspondente canadense, a Canadian Task Force on Preventive Health Care e o American College of Obstetrics and Gynecology não recomendam o rastreamento para câncer de ovário, sob a alegação de que os malefícios dos testes falsos-positivos superam amplamente os benefícios de eventuais diagnósticos precoces ${ }^{26}$.

Devido à posição anatômica do ovário, supunha-se que o aumento de seu volume não causaria sintomas em fases iniciais, ou seja, antes de ser muito grande ou que houvesse focos de disseminação. Entretanto, este conceito do "matador silencioso" tem sido contestado e, ao que parece, deve ser descartado. Mais de 30 estudos demonstraram que mulheres com câncer de ovário, em qualquer estádio, apresentam sintomas até mesmo 36 meses antes do diagnóstico ${ }^{27}$. Estes sintomas são geralmente subestimados pelas mulheres e por seus médicos, por serem inespecíficos e presentes em muitas outras condições benignas e frequentes. Entretanto, ao se examinar uma paciente com mais de 50 anos de idade, especialmente na pós-menopausa, que se queixe repetidamente de constante distensão abdominal, mudanças em hábitos intestinais ou urinários, dor abdominal ou pélvica ou aumento da circunferência abdominal - para a qual seja afastada condição aguda, como gastroenterites etc - deve-se considerar câncer de ovário entre os diagnósticos diferenciais. Esta tem sido a recomendação de várias organizações médicas na América do Norte e Europa. Há dois anos, o então presidente norte-americano George Bush investiu mais de 8 milhões de dólares em uma campanha de educação usando exatamente esta recomendação, dado que mulheres sintomáticas podem ser identificadas pelos seus clínicos e serem submetidas a exames de detecção do câncer de ovário ${ }^{28}$.

É importante, entretanto, entender que esses sintomas são vagos e ainda não totalmente compreendidos. Embora vários grupos estejam tentando estabelecer um índice diagnóstico baseado no conjunto de sintomas clínicos de risco, nenhum desses índices foi testado prospectivamente para rastreamento em grande escala na população geral. Dois ensaios clínicos randomizados conduzidos na Inglaterra tentaram essa estratégia, mas falharam ao identificar como ponto de entrada a consulta com o médico de família ou com um clínico geral ${ }^{29,30}$. Ambos os estudos demonstram, entretanto, que esses médicos não conseguiram mudar sua prática, passando a considerar estes sintomas como possíveis indícios de câncer de ovário.

No Sistema de Saúde da Inglaterra, estabeleceu-se, recentemente, um sistema de referência de urgência em que mulheres com suspeita de certos cânceres seriam examinadas e testadas por um especialista em, no máximo, duas semanas. Este encaminhamento é feito por meio de telefone ou fax, e um dos cânceres alvo é o de ovário. O processo como um todo tem por objetivo final reduzir os prazos para o diagnóstico desses cânceres. Resultados preliminares mostram que, para o câncer de ovário, somente $18 \%$ dos cânceres identificados foram encaminhados com urgência ${ }^{29}$. Antes da implantação deste sistema, pesquisadores britânicos mostraram que $86 \%$ das pacientes com câncer de ovário procuraram um clínico geral e, para estas, o período entre a consulta médica e o diagnóstico foi maior do que para aquelas que procuraram o hospital diretamente ${ }^{30}$.

Alguns grupos de pesquisadores estão atualmente conduzindo estudos que visam estabelecer quais seriam os sintomas específicos do câncer ovariano inicial e qual seria a melhor estratégia para diminuir atrasos no diagnóstico. Até que se demonstre o contrário, a triagem de pacientes sintomáticas parece ser a maneira mais promissora de se detectar o câncer de ovário, enquanto a doença tem um volume menor e é de mais fácil controle.

O diagnóstico de câncer de ovário tem sido uma sentença de óbito e, embora nas últimas décadas tenhamos conseguido regimes terapêuticos capazes de controlar melhor a doença, ainda não foi possível desenvolver um teste capaz de identificar a doença em fase precoce. Ganhamos terreno na prevenção primária com a liberação sexual dos anos 1960 e a adoção do uso de contraceptivos orais, principalmente entre aquelas mulheres de nível social mais alto e, portanto, com maior risco de desenvolvimento da doença. Contudo, outras alternativas precisam ser desenvolvidas. Em relação à prevenção secundária, enquanto não conhecermos melhor a história natural da doença, ficamos limitados a tentar alternativas não ideais. A explosão numérica dos estudos de biologia molecular é alvissareira, porém, dada a complexidade da análise ao nível molecular e sua consequente validação, a identificação de perfis de risco não será obtida em curto prazo. Parece-nos mais promissor o estudo detalhado dos sintomas da doença inicial, sendo que os médicos devem estar alertas para sintomas abdominais inespecíficos em mulheres com mais de 50 anos. 


\section{Referências}

1. Sankaranarayanan R, Ferlay J. Worldwide burden of gynaecological cancer: the size of the problem. Best Pract Res Clin Obstet Gynaecol. 2006;20(2):207-25.

2. Huang L, Cronin KA, Johnson KA, Mariotto AB, Fever EJ. Improved survival time: what can survival cure models tell us about population-based survival improvements in late-stage colorectal, ovarian, and testicular cancer? Cancer. 2008;112(10):2289-300.

3. Chu CS, Rubin SC. Screening for ovarian cancer in the general population. Best Pract Res Clin Obstet Gynaecol. 2006;20(2):307-20.

4. Permuth-Wey J, Sellers TA. Epidemiology of ovarian cancer. Methods Mol Biol. 2009;472:413-37. Review.

5. Rebbeck TR, Kauff ND, Domchek SM. Meta-analysis of risk reduction estimates associated with risk-reducing salpingo-oophorectomy in BRCA 1 or BRCA2 mutation carriers. J Natl Cancer Inst. 2009; 101 (2):80-7.

6. Nossov V, Amneus M, Su F, Lang J, Janco JM, Reddy ST, et al. The early detection of ovarian cancer: from traditional methods to proteomics. Can we really do better than serum CA-125? Am J Obstet Gynecol. 2008;199(3):215-23.

7. Coticchia CM, Yang J, Moses MA. Ovarian cancer biomarkers: current options and future promise. J Natl Compr Canc Netw. 2008;6(8):795-802.

8. Ness RB, Cottreau C. Possible role of ovarian epithelial inflammation in ovarian cancer. J Natl Cancer Inst. 1999;91 (17):1459-67.

9. Hannibal CG, Rossing MA, Wicklund KG, Cushing-Haugen KL. Analgesic drug use and risk of epithelial ovarian cancer. Am J Epidemiol. 2008; 167(12): 1430-7.

10. Wu AH, Pearce CL, Tseng CC, Templeman C, Pike MC. Markers of inflammation and risk of ovarian cancer in Los Angeles County. Int J Cancer. 2009; 124(6):1409-15.

11. Soegaard M, Frederiksen K, Jensen A, Høgdall E, Høgdall C, Blaakaer J, et al. Risk of ovarian cancer in women with first-degree relatives with cancer. Acta Obstet Gynecol Scand. 2009;88(4):449-56.

12. Chen VW, Ruiz B, Killeen JL, Coté TR, Wu XC, Correa CN. Pathology and classification of ovarian tumors. Cancer. 2003;97(10 Suppl):2631-42.

13. Kurman RJ, Shih leM. Pathogenesis of ovarian cancer: lessons from morphology and molecular biology and their clinical implications. Int J Gynecol Pathol. 2008;27(2):151-60.

14. McCluggage WG. My approach to and thoughts on the typing of ovarian carcinomas. Clin Pathol. 2008;61 (2):152-63.

15. Jemal A, Siegel R, Ward E, Hao Y, Xu J, Murray T, et al. Cancer statistics, 2008. CA Cancer J Clin. 2008;58(2):71-96.

16. Heintz AP, Odicino F, Maisonneuve P, Beller U, Benedet JL, Creasman WT, et al. Carcinoma of the ovary. J Epidemiol Biostat. 2001;6(1):107-38.

17. Collaborative Group on Epidemiological Studies of Ovarian Cancer, Beral V, Doll R, Hermon C, Peto R, Reeves G. Ovarian cancer and oral contraceptives: collaborative reanalysis of data from 45 epidemiological studies including 23,257 women with ovarian cancer and 87,303 controls. Lancet. 2008;371(9609):303-14.

18. Franco EL, Duarte-Franco E. Ovarian cancer and oral contraceptives. Lancet. 2008;371(9609):277-8.

19. Shuster LT, Gostout BS, Grossardt BR, Rocca WA. Prophylactic ooforectomy in premenopausal women and long-term health. Menopause Int. $2008 ; 14(3): 111-6$.

20. Evans GR, Gaarenstroom KN, Stirling D, Shenton A, Maehle L, Dørum A, et al. Screening for Familial Ovarian Cancer: Poor survival of BRCA 1/2 related cancers. J Med Genet. In press 2008.

21. Jacobs IJ, Menon U. Progress and challenges in screening for early detection of ovarian cancer. Mol Cell Proteomics. 2004;3(4):355-66.

22. Van Holsbeke C, Van Calster B, Testa AC, Domali E, Lu C, Van Huffel S, et al. Prospective internal validation of mathematical models to predict malignancy in adnexal masses: results from the international ovarian tumor analysis study. Clin Cancer Res. 2009; 15(2):684-91.

23. Jacobs IJ, Skates SJ, MacDonald N, Menon U, Rosenthal AN, Davies AP, et al. Screening for ovarian cancer: a pilot randomised controlled trial. Lancet. 1999;353(9160):1207-10.

24. Buys SS, Partridge E, Greene MH, Prorok PC, Reding D, Riley TL, et al. Ovarian cancer screening in the Prostate, Lung, Colorectal and Ovarian (PLCO) cancer screening trial: findings from the initial screen of a randomized trial. Am J Obstet Gynecol. 2005; 193(5):1630-9.

25. Menon U, Gentry-Maharaj A, Ryan A, Sharma A, Burnell M, Hallett R, et al. Recruitment to multicentre trials-lessons from UKCTOCS: descriptive study. BM. 2008;337:a2079.

26. Franco EL, Duarte-Franco E, Rohan TE. Evidence-based policy recommendations on cancer screening and prevention. Cancer Detect Prev. 2002;26(5):350-61.

27. Bankhead CR, Kehoe ST, Austoker J. Symptoms associated with diagnosis of ovarian cancer: a systematic review. BJOG. 2005; 1 12(7):857-65.

28. Ovarian Cancer National Alliance. Johanna's Law: the gynecologic cancer education and awareness act of 2007 [Internet]. Washington, DC, 2009 [cited 2009 Mar 20]. Available from: http://www.ovariancancer.org/index.cfm?fuseaction=Page.viewPage\&pageld=627\&parentID $=525 \&$ grandparent $\mid \mathrm{D}=4$ \&node $\mathrm{I}=1$

29. Neal RD, Allgar VL, Ali N, Leese B, Heywood P, Proctor G, et al. Stage, survival and delays in lung, colorectal, prostate and ovarian cancer: comparison between diagnostic routes. Br J Gen Pract. 2007;57(536):212-9.

30. Allgar VL, Neal RD. Delays in the diagnosis of six cancers: analysis of data from the National Survey of NHS Patients: Cancer. Br J Cancer. $2005 ; 92(11): 1959-70$. 